\title{
Human psychopharmacology and dose-effects of salvinorin A, a kappa-opioid agonist hallucinogen present in the plant Salvia divinorum
}

\author{
Matthew W. Johnson ${ }^{1}$, Katherine A. MacLean ${ }^{1}$, Chad J. Reissig ${ }^{1}$, Thomas E. Prisinzano², \\ and Roland R. Griffiths ${ }^{1,3}$ \\ 1 Department of Psychiatry and Behavioral Sciences, Johns Hopkins University School of \\ Medicine, Baltimore, MD 21224-6823, USA \\ 2 Department of Medicinal Chemistry, The University of Kansas, Lawrence, KS, 66045-7582, \\ USA \\ ${ }^{3}$ Department of Neuroscience, Johns Hopkins University School of Medicine, Baltimore, MD \\ 21224-6823, USA
}

\begin{abstract}
Salvinorin A is a potent, selective nonnitrogenous kappa opioid agonist and the known psychoactive constituent of Salvia divinorum, a member of the mint family that has been used for centuries by Mazatec shamans of Mexico for divination and spiritual healing. Salvia divinorum has over the last several years gained increased popularity as a recreational drug. This is a doubleblind, placebo controlled study of salvinorin A in 4 psychologically and physically healthy hallucinogen-using adults. Across sessions, participants inhaled 16 ascending doses of salvinorin A and 4 intermixed placebo doses under comfortable and supportive conditions. Doses ranged from $0.375 \mu \mathrm{g} / \mathrm{kg}$ to $21 \mu \mathrm{g} / \mathrm{kg}$. Subject-rated drug strength was assessed every 2 minutes for 60 minutes after inhalation. Orderly time- and dose-related effects were observed. Drug strength ratings peaked at 2 minutes (first time point) and definite subjective effects were no longer present at approximately 20 minutes after inhalation. Dose-related increases were observed on questionnaire measures of mystical-type experience (Mysticism Scale) and subjective effects associated with classic serotonergic $(5-\mathrm{HT} 2 \mathrm{~A}$ ) hallucinogens (Hallucinogen Rating Scale). Salvinorin A did not significantly increase heart rate or blood pressure. Participant narratives indicated intense experiences characterized by disruptions in vestibular and interoceptive signals (e.g., change in spatial orientation, pressure on the body) and unusual and sometimes recurring themes across sessions such as revisiting childhood memories, cartoon-like imagery, and contact with entities. Under these prepared and supportive conditions, salvinorin A occasioned a unique profile of subjective effects having similarities to classic hallucinogens, including mystical-type effects.
\end{abstract}

\section{Keywords}

salvinorin A; Salvia divinorum; kappa opioid agonist; hallucinogen; psychedelic; humans

Corresponding Author: Matthew W. Johnson, Behavioral Pharmacology Research Unit, Department of Psychiatry and Behavioral Sciences, Johns Hopkins University School of Medicine, 5510 Nathan Shock Drive, Baltimore, MD 21224-6823, Tel: 410-550-0056, Fax: 410-550-0030,mwj@jhu.edu.

Publisher's Disclaimer: This is a PDF file of an unedited manuscript that has been accepted for publication. As a service to our customers we are providing this early version of the manuscript. The manuscript will undergo copyediting, typesetting, and review of the resulting proof before it is published in its final citable form. Please note that during the production process errors may be discovered which could affect the content, and all legal disclaimers that apply to the journal pertain. 


\section{Introduction}

Shamans of the Mazatec people of Oaxaca, Mexico have used Salvia divinorum, a member of the mint family, for at least centuries in ethnomedical practices including divination and spiritual healing (Valdes, 1994; Ott, 1995, 1996; Siebert, 1994). S. divinorum contains the known psychoactive constituent salvinorin $\mathrm{A}$, which is a neoclerodane diterpene and a potent nonnitrogenous kappa opioid agonist, with no activity at the 5- $\mathrm{HT}_{2 \mathrm{~A}}$ serotonin receptor, the principal site of activity of classic hallucinogens such as LSD, psilocybin, dimethyltryptamine, and mescaline (Roth et al., 2002; Prisinzano, 2005). Studies in monkeys show salvinorin A produces discriminative stimulus effects similar to other high efficacy kappa agonists (Butelman et al., 2004). A kappa-like profile of antinociceptive and behavioral effects has also been demonstrated in rodents (Fantegrossi et al., 2005; Wang et al., 2005; Zhang et al., 2005; McCurdy et al., 2006; Carlezon et al., 2006). Nonhuman research with salvinorin A has shown mixed results regarding abuse potential. Evidence suggestive of no or low abuse potential includes research showing salvinorin A to elevate thresholds for intracranial stimulation and decrease extracellular dopamine concentrations in the nucleus accumbens in rats (Carlezon et al., 2006), and produce conditioned place aversion in mice (Zhang et al., 2005). Evidence suggestive of abuse potential are recent findings demonstrating intracerebroventricular salvinorin A self-administration and conditioned place preference in mice at relatively low doses (Braida et al., 2008).

Although the ethnomedical use of S. divinorum by shamans dates back at least for hundreds of years, an understanding of the psychoactive effects of salvinorin A by American and European drug users dates back only about 15 years (Siebert, 1994; Ott, 1995). In traditional Mexican use, the leaves of S. divinorum are chewed or made into an infusion and swallowed (Valdes, 1994; Siebert, 1994, Ott, 1995). Outside the Mazatec, drug users typically inhale the drug via smoking or, less frequently, via volatilization, although buccal administration also occurs (Baggott et al., 2010; González et al., 2006). Internet vendors and "head shops" sell $S$. divinorum plants, dried leaves, leaf extracts with increased salvinorin A concentrations (for smoking), and tinctures (for buccal administration). As of 2006 at least 1.8 million people age 12 or older have used the drug in the United States (Office of Applied Studies, 2007), and the prevalence is likely higher now. Survey research suggests that effects are brief and that users claim positive after-effects such as increased insight and improved mood (Baggott et al., 2010). One study found high scores on a measure of state anxiety when asking participants to retrospectively evaluate their last use (González et al., 2006). During a recent 10-year period, the California Poison Control System received 37 telephone calls concerning S. divinorum exposures with reported neurologic, cardiovascular, and gastrointestinal effects (Vohra et al, 2009). Three cases reported that $S$. divinorum use was associated with an extended psychotic-type reaction, although concurrent other drug use (Singh, 2007), suspected schizophrenic predisposition (Przekop and Lee, 2009), and injuries secondary to medical treatment (Paulzen and Grunder, 2008) have complicated interpretation of these cases. Possession or use of $S$. divinorum is currently illegal in 13 nations and 15 states within the United States, and additional nations and states have implemented lesser forms of control.

Given the wide availability, continued popular use, and legal controversy, information is needed regarding the human psychopharmacology of salvinorin A. In addition to providing information about this novel drug, studying the effects salvinorin A may assist in identifying new opioid receptor modulators that may have therapeutic applications in certain psychiatric disorders (e.g., Alzheimer's disease, schizophrenia, bipolar disorder, cocaine abuse) and in the treatment of pain (Mello and Negus, 2000; Sheffler and Roth, 2003; Kivell and Prisinzano, 2010). This is a report of preliminary findings of basic physiological, behavioral 
and subjective effects of inhaled salvinorin A across a range of doses, from sub-threshold to high, delivered under comfortable and interpersonally supportive conditions to healthy participants who reported histories of hallucinogen use.

\section{Methods}

\subsection{Participants}

Participants were recruited from the Baltimore, MD area through flyers and ads in local newspapers. After a phone screen confirmed basic inclusion criteria (age, high-school-level education, and prior hallucinogen use), volunteers came to the laboratory for in-person screening. A social worker (who was trained by psychometricians and had a psychiatrist and clinical psychologist available for consultation) conducted a structured clinical interview to exclude individuals with a current or past history of meeting DSM-IV criteria for schizophrenia; psychotic disorder (unless substance-induced or due to a medical condition); bipolar I or II disorder; alcohol or drug dependence (excluding caffeine and nicotine) for the last 5 years; severe major depression; dissociative disorder; and other psychiatric conditions judged to be incompatible with establishment of rapport or safe exposure to hallucinogens. Participants were excluded who reported having a first or second degree relative with schizophrenia, psychotic disorder (unless substance induced or due to a medical condition), or bipolar I or II disorder. Medical evaluation included a physical exam conducted by a nurse practitioner, an ECG evaluated by a physician, and a physician evaluation of blood sample analyses that included a complete blood count with differential and a chemistry panel including electrolytes, glucose, urea, creatinine, uric acid, liver profile, total cholesterol, and triglycerides. Additional screening visits assessed general motivation for participation and established trust and rapport with the study staff. Participants agreed to refrain from using $S$. divinorum or illicit drugs during the course of the study.

Four participants ( $M=29.5$ years old, range $=23-35$ years old; 2 females $)$ passed screening and completed all drug sessions and data collection (Note: One additional individual participated in pilot sessions to develop and refine the inhalation procedure; these data are not included in the reported analyses). Participant bodyweights were 58.5, 74.6, 64.4 , and $67.1 \mathrm{~kg}$ for the two males and two females, respectively. Participants had at least a high-school level of education ( $M=15$ years, range $=14-16$ years) reported using $S$. divinorum at least once in the past five years ( $M=16$ uses, range $=2-40$ uses). Participants were compensated $\$ 50$ per session (plus a bonus of $\$ 50$ per session upon completion of data collection). Participations were also motivated by their reported interest in spirituality and/or altered states of consciousness. Transportation to and from the study site (via taxi) was provided for all participants. The Institutional Review Board of the Johns Hopkins University School of Medicine approved the study, and all participants gave their informed consent before participation.

\subsection{Drug preparation and delivery device}

Salvinorin A was isolated from S. divinorum leaves and shown to be $99.5 \%$ pure by HPLC. Because weighing the very small doses of salvinorin A required for this study is difficult, a larger quantity was weighed on an analytical balance and then dissolved in a measured volume of HPLC grade acetone. After filtering through a sterilized, acetone-approved 0.2 $\mu \mathrm{m}$ filter (Millex-LG Filter Unit, Millipore Corporation, Billerica, MA), $1 \mathrm{ml}$ of solution was placed into a $5 \mathrm{ml}$ round bottom chemistry flask and allowed to evaporate, leaving the dose of salvinorin A as a residue on the interior surface of the flask. Before the session, the flask was attached to the bottom of a chemistry vacuum adapter by ground glass tapered joint and secured by a metal Keck clip. A vinyl tube ( $23 \mathrm{~cm}$ long, $6.35 \mathrm{~mm}$ inside diameter) was connected to the vacuum line of the adapter for inhalation. The tubing was replaced 
between participants but was not replaced for different sessions for the same participant. The resulting "pipe" was held stable by a ring stand clamp during administration, and the tube extended through a hole in a room divider toward the participant's sitting area.

\subsection{Drug administration}

Sixteen doses of salvinorin A were administered in an ascending order. Although it is unknown whether salvinorin A effects are bodyweight dependent, doses in this study were adjusted for participant bodyweight based on knowledge that some but not all psychoactive drug effects are bodyweight dependent. The lowest dose in the sequence was $0.375 \mu \mathrm{g} / \mathrm{kg}$ of bodyweight and subsequent doses were $0.75 \mu \mathrm{g} / \mathrm{kg}, 1.5 \mu \mathrm{g} / \mathrm{kg}$, and thereafter increased by an increment of $1.5 \mu \mathrm{g} / \mathrm{kg}$ until the maximum dose of $21 \mu \mathrm{g} / \mathrm{kg}$ was reached. Four placebo doses were inserted in the ascending sequence of salvinorin A doses such that each consecutive block of 5 sessions included 1 placebo. The position of the single placebo within each block of 5 sessions was determined randomly for each volunteer. Participants were told that on any session they may receive a dose of salvinorin A or placebo but were not told about the ascending design or frequency of placebos.

For each session the round-bottom flask was heated from underneath with a butane microtorch with approximate flame temperature of $1,700{ }^{\circ} \mathrm{C}$ (model ST200T, Bernzomatic, Huntersville, NC). Although the boiling point and combustion temperature of salvinorin A have not been empirically determined, the flame temperature was assumed to be sufficient to heat the salvinorin $\mathrm{A}$ on the interior of the flask to its boiling point during the decreased air pressure created by inhalation, without substantial combustion of salvinorin A. The microtorch was adjusted so that the flame was approximately $1.5 \mathrm{~cm}$ in length, and the tip of the flame was held in contact with the round-bottom flask. Using these procedures, inhalation of un-volatilized drug was unlikely because the salvinorin A residue left upon evaporation of acetone appeared strongly attached to the interior of the flask, and in tests with various rates of mechanical airflow (simulated inhalation) without heat, salvinorin A was observed to remain undisturbed. Tests with mechanical airflow and the application of the microtorch showed the method to result in the volatilization of salvinorin A with little scorching (small black marks presumably resulting from combustion). Seated in a comfortable reclining lounger, the participant inhaled slowly for $40 \mathrm{~s}$ while the flask was heated, followed by a verbally cued exhale. For placebo sessions, the same protocol was followed but no salvinorin A was in the flask. The unblinded session monitor who heated the flask was obscured from the participant's view (behind the divider), while the blinded staff member collected drug-strength ratings and all other pre- and post-session data from the participant.

\subsection{Drug sessions}

Each participant completed 20 sessions across several weeks (range $=8-14$ weeks). Participants typically completed $1-3$ sessions per week with consecutive sessions separated by at least 1 day (range $=1$ day to 4.5 weeks).

Each meeting at the study site lasted approximately 2.5 hours. Upon arrival, participants provided an expired air sample that was required to indicate a blood alcohol concentration of $0.00 \%$ for the session to continue (Alco-Sensor IV, Intoximeters, Inc., Saint Louis, MO). In addition, abstinence from opioids, cocaine, and benzodiazepines was confirmed by urine test before each session (model MUI-PM, Medimpex United Inc., Bensalem, PA). This urine test panel was selected because it can detect opioids such as oxycodone and buprenorphine with kappa opioid effects. Abstinence from hallucinogens (and other drugs not listed above) was not biologically confirmed. However, participants consented to have their urine tested for "various drugs" and were not informed about which drugs were included in the urine test. 
Female participants were required to provide a negative urine pregnancy test before each session (model HCG-001, Medimpex United Inc., Bensalem, PA). Also before drug administration, participants briefly reviewed experiences from the last session with the study staff. During drug administration, participants were seated in a comfortable semi-upright or reclined position and wore eyeshades for 3-5 minutes before and for 10-30 minutes after drug administration. A relaxing instrumental music track (Steven Halpern, "Awakening", Music for Sound Healing, Inner Peace Music, 1999) was played throughout the session as a continuous loop. Approximately 1 hour after drug administration participants completed several questionnaires and computer-based assessments (duration $=45-60$ minutes).

\subsection{Measures}

2.5.1 Physiology-Blood pressure (systolic and diastolic using oscillometric method with the blood pressure cuff placed on the arm) and heart rate were monitored using a NonInvasive Patient Monitor Model 507E (Criticare Systems, Waukesha, WI). Data were collected at baseline (for at least 5 minutes before drug administration) and every 2 minutes during the 60 minutes following drug administration.

2.5.2 Timecourse-Participants indicated overall drug strength using an 11-point scale (0 = definitely no effect; 1 or $2=$ possible salvinorin A effects; $3-10=$ definite salvinorin A effects with 10 representing "the strongest effect imaginable for salvinorin A"). Participants were verbally cued to provide a rating of drug strength at baseline and every 2 minutes for 60 minutes after drug administration. In the case of missing reports (i.e., participant unresponsive during the first few minutes of drug effects), the value of ' 10 ' was imputed.

2.5.3 Safety and tolerability-Although not reported by users in retrospective analyses (Baggott et al., 2010; González et al., 2006), tremor has been observed in rhesus monkeys at a substantially higher dose $(100 \mu \mathrm{g} / \mathrm{kg}$ ) than our maximum dose (Butelman; personal communication, 2006). The Fahn-Tolosa-Marin Tremor Rating Scale (TRS; Fahn et al., 1993) was used to classify resting and kinetic tremor severity on a 5-point scale. At baseline and at 15 and 30 minutes after drug administration, the study staff visually assessed kinetic tremor intensity as the participant bent both of his or her extended arms to touch middle finger to nose. Study staff also rated the magnitude of resting tremor during the time interval leading up to the assessments of kinetic tremor (i.e., 0-15 $\mathrm{min}, 15-30 \mathrm{~min}$ ) by closely observing the participant.

To assess psychological effects that might warrant discontinuing future sessions, participants were asked at the end of each session "Would you absolutely refuse to receive the same or higher dose of today's drug in future sessions?" A "no" response was required to continue. In addition, study staff interviewed the participant before and after each session to determine how $\mathrm{s} / \mathrm{he}$ felt about continuing to participate.

2.5.4 End-of-session subject-rated effects-Shortly after the 60-minute time point, participants were asked to retrospectively rate the strength of the peak drug effect during the session using the same 11-point scale used to assess the time course of drug effects. Because kappa opioid agonists, including salvinorin A, have been reported to induce dysphoric effects (Walsh et al., 2001; González et al., 2006), participants were asked to complete retrospective ratings of "drug liking" and "drug disliking" ( 2 questions; $0=$ neutral or no effect, $1=$ like/dislike but not very much, $2=$ like/dislike somewhat, $3=$ like/dislike quite a bit, $4=$ like/dislike very much), and "good effects" and "bad effects" ( 2 questions; $0=$ no good/ bad effects at all, 1= a little, 2= moderately, $3=$ quite a bit, $4=$ very much). 
Consistent with case reports and survey studies of S. divinorum users (Siebert, 1994; Baggott et al., 2010; Bücheler et al., 2005; González et al., 2006) we hypothesized that salvinorin A would elicit subjective effects with substantial overlap with classic hallucinogens (e.g., psilocybin, LSD). We further hypothesized that, unlike orally administered psilocybin and LSD, volatilized and inhaled salvinorin A would have a timecourse profile more similar to intravenous dimethyltryptamine (Strassman et al., 1994). In addition, because $S$. divinorum has been used by shamans for divination and "visionary effects" (Ott, 1995), we hypothesized that salvinorin A might elicit subject-rated mysticaltype effects. We used two questionnaires to assess these effects. The Hallucinogen Rating Scale (HRS; 99 items) was designed to show sensitivity to dimethyltryptamine (Strassman et al., 1994) and has also been shown sensitive to psilocybin (Griffiths et al., 2006). It consists of six subscales assessing various aspects of hallucinogen effects: Intensity (effects related to the strength of the overall experience), Somaesthesia (interoceptive, visceral and tactile effects), Affect (emotional and affective responses), Perception (visual, auditory, gustatory, and olfactory effects), Cognition (alterations in thought processes or content), and Volition (awareness of the environment and capacity to willfully control one's body). Most items are rated on a 5-point scale ( $0=$ not at all; $4=$ extremely). The Mysticism Scale (32 items) was developed to assess primary mystical or spiritual experiences (Hood et al., 2001) and has been shown to be sensitive to the effects of psilocybin (Griffiths et al., 2006). Each item is rated on a 9-point scale $(-4=$ this description is extremely not true of my own experience; 0 $=\mathrm{I}$ cannot decide $+4=$ this description is extremely true of my own experience).

Approximately 1 hour after drug administration, participants completed the HRS and Mysticism Scale with reference to their experiences since receiving the inhaled drug.

\subsection{Analysis}

Subject-rated drug strength ratings collected throughout the timecourse were used to calculate, for individual participant, area under the curve (AUC) values using the trapezoidal rule. Repeated measures analysis of variance (ANOVA) with within-subjects factor of dose (17 dose levels including placebo) was used to examine dose-related changes in the subjectrated measures and physiology (peak heart rate and blood pressure). For each participant, drug strength ratings across the 4 placebo sessions were averaged to create a single placebo data point. Tukey's HSD tests were conducted to compare dose levels, with family-wise error rate maintained at $\alpha=.05$.

\section{Results}

\subsection{Time Course of Acute Drug Effects}

Orderly time- and dose-related effects were observed. Subject-rated drug strength peaked at 2 minutes (first time point) and then progressively decreased toward pre-inhalation levels. By 20 minutes after inhalation, mean ratings indicated only a "possible mild" effect (see Figure 1a). Two participants (1 male, 1 female) provided the maximal rating of 10 at one or more time points during at least one session. Both male participants were unresponsive (scores imputed as 10) on at least one time point for at least one dose. Specifically, one was unresponsive at the 2-minute time point in the $15,16.5,18$, and $21 \mu \mathrm{g} / \mathrm{kg}$ conditions and the 4-minute time point in the $21 \mu \mathrm{g} / \mathrm{kg}$ condition. The other male was unresponsive at the 2minute time point in the 18 and $19.5 \mu \mathrm{g} / \mathrm{kg}$ conditions.

\subsection{End-of-session retrospective self-reports}

The effect of dose on peak drug strength (end-of-session rating) was significant $(F(16,48)=$ $12.6, p<.001$ ), and post-hoc tests confirmed that all but one dose in the range $4.5 \mu \mathrm{g} / \mathrm{kg}$ to $21 \mu \mathrm{g} / \mathrm{kg}$ were rated significantly stronger than placebo (see Figure 1b). Results were similar when conducted with AUC values for drug strength $(F(16,48)=4.5, p<.001)$. However, 
likely due to inter-participant variability in duration of drug effects, post-hoc tests showed only the highest two doses were significantly stronger than placebo. End-of-day ratings of drug liking and good effects followed a similar dose-related pattern as ratings of overall drug strength, and the effect of dose was significant for both liking $(F(16,48)=7.5, \mathrm{p}<.001)$ and good effects $(F(16,48)=4.4, \mathrm{p}<.001)$. Mean ratings of drug disliking and bad effects were low $(M \mathrm{~s}<1)$ across all sessions, and there was no significant effect of dose for either measure ( $p s>.30)$. Mean ratings of the highest dose indicated generally positive effects and a lack of dysphoric effects: liking $M=3$ (range $=2-4$ ); good effects $M=2.25$ (range=1-4); disliking $M=.5$ (range $=0-2$ ); and bad effects $M=.5$ (range $=0-1$ ), with only 1 participant indicating "disliked somewhat." There were significant effects of dose on all subscales of the HRS and on the M Scale $(p<.05)$ (Table 1). Post-hoc tests showed significant increases (vs. placebo) on HRS-Intensity beginning at a dose of $4.5 \mu \mathrm{g} / \mathrm{kg}$, which is consistent with the pattern observed for drug strength (see Figure 1b). The remaining HRS subscales were significantly greater than placebo only at the highest dose levels (Cognition, Affect, Somaesthesia, and Perception) or not at all (Volition). Scores on the M Scale were significantly greater than placebo at the three highest dose levels (Table 1).

\subsection{Safety and Tolerability}

The effect of dose on blood pressure and heart rate were not significant $(p>.05)$, indicating no dose-related change in these physiological measures (see Figure 1c). No resting or kinetic tremors were observed during any session (TRS rating $=0$ for all data points). No participant refused to receive the same or higher dose at the end of any session. These results suggest that inhaled salvinorin A was physiologically safe and psychologically well tolerated across the range of doses tested.

\section{Discussion}

Consistent with results from nonhuman animal research (Mowry et al. 2003), the present results suggest a safe physiological profile for salvinorin A at the studied doses, under controlled conditions, and in psychologically and physically healthy hallucinogenexperienced participants. Salvinorin A produced no significant changes in heart rate or blood pressure; no tremor was observed; and no adverse events were reported. Participants tolerated all doses. However, because of the small sample and the healthy, hallucinogenexperienced status of participants, conclusions regarding safety are limited.

Salvinorin A resulted in orderly dose- and time-related participant ratings of drug strength. In psychopharmacology studies with drug experienced volunteers, it is quite unusual for volunteers to report drug strength ratings as high as those observed in the present study, with 2 subjects providing ratings of 10 , defined as "as strong as imaginable for this drug." The range of doses examined in this study will be useful for selecting doses for future studies using the same administration procedures (e.g., 3, 7.5, and $21 \mu \mathrm{g} / \mathrm{kg}$ for low, medium, and high doses, respectively). Given the increasing trend and lack of plateau in drug strength ratings (see Figure 1b) and the lack of adverse effects in this study, future studies may also cautiously explore higher doses of salvinorin A. The relative lack of dysphoric effects ("disliking" and "bad effects") and presence of dose-related positive effects ("liking" and "good effects") contrasts with previous research with kappa agonists (Walsh et al., 2001) and S. divinorum (González et al., 2006) showing mainly dysphoric effects. This difference with previous research may relate to participant experience with, expectation of, and preparation for strong altered states of consciousness. In addition, salvinorin A produced dose-related increases on most subscales of the Hallucinogen Rating Scale and on the Mysticism Scale. Although limited by cross-study comparisons, high-dose hallucinogentype effects as measured by the HRS appeared similar to those produced by intravenous dimethyltryptamine (Strassman et al., 1994) and oral psilocybin (Griffiths et al., 2006), but 
with qualitative differences (i.e., lower magnitude on perceptual and cognition scales). In addition, the mystical-type effects observed at the highest doses of salvinorin A appeared similar in magnitude to previous results with high-dose oral psilocybin (Griffiths et al., 2006).

The volatilization and inhalation procedures had limitations, such as a lack of quantitative temperature control, which could have possibly resulted in incomplete volatilization or combustion of salvinorin A. In addition, not replacing the tubing between sessions could have theoretically allowed for depositing and re-volatilization of salvinorin A across sessions. However, salvinorin A was observed to virtually completely volatilize with little scorching, and this outcome may be consistent with usual use because combustion also occurs in the smoking of $S$. divinorum, the most common route of use. Also, a post-hoc t-test comparing peak drug strength between the first and final placebo sessions failed to show a significant difference $(\mathrm{p}=.18)$, suggesting that depositing and re-volatilization did not likely affect results. Ultimately, the orderly dose-related effects shown in Figure 1a and Figure 1b suggest reasonable precision in the administration procedures.

In the comfortable and supportive conditions of the current study (similar to those recommended for human research with classic hallucinogens; Johnson et al., 2008), salvinorin A occasioned a unique profile of subjective effects having similarities to classic hallucinogens, including mystical-type effects. Although participant narratives indicated intense, highly unusual experiences characterized by changes in spatial orientation, feelings of energy or pressure on different parts of the body, and unusual and sometimes recurring themes across sessions such as revisiting childhood memories, cartoon-like imagery and contact with entities, participants were largely behaviorally inactive. In this regard, participant experiences differed notably from some of the more chaotic effects observed in online videos of $S$. divinorum use that have gained notoriety (Lange et al., 2010). In conclusion, these results suggest the feasibility of future research examining the effects of salvinorin A, and potentially other high efficacy kappa agonists, in healthy hallucinogenexperienced participants.

\section{References}

Baggott MJ, Erowid E, Erowid F, Galloway GP, Mendelson J. Use patterns and self-reported effects of Salvia divinorum: An internet-based survey. Drug Alcohol Depend. 2010; 111:250-6. [PubMed: 20627425]

Braida D, Limonta V, Capurro V, Fadda P, Rubino T, Mascia P, Zani A, Gori E, Fratta W, Parolaro D, Sala M. Involvement of kappa-opioid and endocannabinoid system on Salvinorin A-induced reward. Biol Psychiatry. 2008; 63:286-92. [PubMed: 17920565]

Bücheler R, Gleiter CH, Schwoerer P, Gaertner I. Use of nonprohibited hallucinogenic plants: increasing relevance for public health? A case report and literature review on the consumption of Salvia divinorum (Diviner's Sage). Pharmacopsychiatry. 2005; 38:1-5. [PubMed: 15706458]

Butelman ER, Harris TJ, Kreek MJ. The plant-derived hallucinogen, salvinorin A, produces kappaopioid agonist-like discriminative effects in rhesus monkeys. Psychopharmacology. 2004; 172:220224. [PubMed: 14586540]

Carlezon WA Jr, Beguin C, DiNieri JA, Baumann MH, Richards MR, Todtenkopf MS, Rothman RB, Ma Z, Lee DY, Cohen BM. Depressive-like effects of the kappa-opioid receptor agonist salvinorin A on behavior and neurochemistry in rats. J Pharmacol Exp Ther. 2006; 316:440-447. [PubMed: 16223871]

Fahn, S.; Tolosa, E.; Marín, C. Clinical rating scale for tremor. In: Jankovic, J.; Tolosa, E., editors. Parkinson's disease and movement disorders. Williams \& Wilkins; Baltimore: 1993. p. 225-234.

Fantegrossi WE, Kugle KM, Valdes LJ 3rd, Koreeda M, Woods JH. Kappa-opioid receptor-mediated effects of the plant-derived hallucinogen, salvinorin A, on inverted screen performance in the mouse. Behav Pharmacol. 2005; 16:627-633. [PubMed: 16286814] 
González D, Riba J, Bouso JC, Gomez-Jarabo G, Barbanoj MJ. Pattern of use and subjective effects of Salvia divinorum among recreational users. Drug Alcohol Depend. 2006; 85:157-62. [PubMed: 16720081]

Griffiths RR, Richards WA, McCann U, Jesse R. Psilocybin can occasion mystical-type experiences having substantial and sustained personal meaning and spiritual significance. Psychopharmacology. 2006; 187:268-283. [PubMed: 16826400]

Hood RW Jr, Ghorbani N, Watson PJ, Ghramaleki AF, Bing MN, Davison HK, Morris RJ, Williamson WP. Dimensions of the mysticism scale: confirming the three-factor structure in the United States and Iran. J Sci Study Relig. 2001; 40:691-705.

Johnson MW, Richards WA, Griffiths RR. Human hallucinogen research: Guidelines for safety. J Psychopharmacol. 2008; 22:603-620. [PubMed: 18593734]

Kivell B, Prisinzano TE. Kappa opioids and the modulation of pain. Psychopharmacology. 2010; 210:109-119. [PubMed: 20372880]

Lange JE, Daniel J, Homer K, Reed MB, Clapp JD. Salvia divinorum: effects and use among YouTube users. Drug Alcohol Depend. 2010; 108:138-40. [PubMed: 20031341]

McCurdy CR, Sufka KJ, Smith GH, Warnick JE, Nieto MJ. Antinociceptive profile of salvinorin A, a structurally unique kappa opioid receptor agonist. Pharmacol Biochem Behav. 2006; 83:109-113. [PubMed: 16434091]

Mello NK, Negus SS. Interactions between kappa opioid agonists and cocaine: preclinical studies. Ann NY Acad Sci. 2000; 909:104-32. [PubMed: 10911926]

Mowry M, Mosher M, Briner W. Acute physiologic and chronic histologic changes in rats and mice exposed to the unique hallucinogen salvinorin A. J Psychoactive Drugs. 2003; 35:379-82. [PubMed: 14621136]

Ott J. Ethnopharmacognosy and human pharmacology of Salvia divinorum and salvinorin A. Curare. $1995 ; 18: 103-129$.

Ott, J. Pharmacotheon: entheogenic drugs, their plant sources and history. 2. Natural Products Co; Kennewick, WA: 1996.

Fahn, S.; Tolosa, E.; Marín, C. Clinical rating scale for tremor. In: Jankovic, J.; Tolosa, E., editors. Parkinson's disease and movement disorders. Williams \& Wilkins; Baltimore: 1993. p. 225-234.

Paulzen M, Gründer G. Toxic psychosis after intake of the hallucinogen salvinorin A. J Clin Psychiatry. 2008; 69:1501-2. [PubMed: 19193352]

Prisinzano TE. Psychopharmacology of the hallucinogenic sage salvia divinorum. Life Sci. 2005; 78:527-531. [PubMed: 16213533]

Przekop P, Lee T. Persistent psychosis associated with Salvia divinorum use. Am J Psychiatry. 2009; 166:7.

Roth BL, Baner K, Westkaemper R, Siebert D, Rice KC, Steinberg S, Ernsberger P, Rothman RB. Salvinorin A: A potent naturally occurring nonnitrogenous kappa opioid selective agonist. Proc Natl Acad Sci USA. 2002; 99:11934-11939. [PubMed: 12192085]

Sheffler DJ, Roth BL. Salvinorin A: The "magic mint" hallucinogen finds a molecular target in the kappa opioid receptor. Trends Pharmacol Sci. 2003; 24:107-109. [PubMed: 12628350]

Siebert DJ. Salvia divinorum and salvinorin A: New pharmacologic findings. J Ethnopharmacol. 1994; 43:53-56. [PubMed: 7526076]

Singh S. Adolescent salvia substance abuse. Addiction. 2007; 102:823-4. [PubMed: 17493110]

Strassman RJ, Qualls CR, Uhlenhuth EH, Kellner R. Dose-response study of N,N-dimethyltryptamine in humans. II. Subjective effects and preliminary results of a new rating scale. Arch Gen Psychiatry. 1994; 51:98-108. [PubMed: 8297217]

Valdes LJ 3rd. Salvia divinorum and the unique diterpene hallucinogen, salvinorin (divinorin) A. J Psychoactive Drugs. 1994; 26:277-283. [PubMed: 7844657]

Vohra R, Seefeld A, Cantrell FL, Clark RF. Salvia divinorum: exposures reported to a statewide poison control system over 10 years. J Emerg Med. 2009 Sep 16. [Epub ahead of print].

Walsh SL, Strain EC, Abreu ME, Bigelow GE. Enadoline, a selective kappa opioid agonist: comparison with butorphanol and hydromorphone in humans. Psychopharmacology. 2001; 157:151-162. [PubMed: 11594439] 
Wang Y, Tang K, Inan S, Siebert D, Holzgrabe U, Lee DY, Huang P, Li JG, Cowan A, Liu-Chen LY. Comparison of pharmacological activities of three distinct kappa ligands (salvinorin A, TRK-820 and 3FLB) on kappa opioid receptors in vitro and their antipruritic and antinociceptive activities in vivo. J Pharmacol Exp Ther. 2005; 312:220-230. [PubMed: 15383632]

Zhang Y, Butelman ER, Schlussman SD, Ho A, Kreek MJ. Effects of the plant-derived hallucinogen salvinorin A on basal dopamine levels in the caudate putamen and in a conditioned place aversion assay in mice: Agonist actions at kappa opioid receptors. Psychopharmacology. 2005; 179:551558. [PubMed: 15682306] 


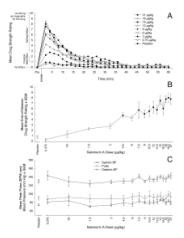

Figure 1. Time- and dose-related changes in drug effects

(A) Subject-rated drug strength (reported at 2-minute intervals) as a function of time (predrug baseline to 60 minutes following inhalation). Data points show mean ratings $(N=4)$. For graphical clarity, alternating doses in the dose sequence were deleted.

(B) Subject-rated peak drug strength from the end-of-session ratings as a function of dose (logarithmic abscissa). Data points show mean ratings $(N=4)$; brackets show + and -1 SEM. Filled symbols indicate that the data point was significantly different from placebo (Tukey's HSD test, $\mathrm{p}<.05)$.

(C) Peak heart rate (beats per minute) and blood pressure ( $\mathrm{mm}$ of $\mathrm{Hg}$ ) as a function of dose (logarithmic abscissa). Data points show means $(\mathrm{N}=4)$; brackets show + and - 1 SEM. 
